\section{Case Reports in Oncology}

\title{
Treatment of Sinonasal Undifferentiated Carcinoma with TPF and Concurrent Chemo- Radiation: Case Report and Literature Review
}

\author{
Emily Barber $^{\mathrm{a}} \quad$ Ann Eapen $^{\mathrm{a}} \quad$ Rupali Nabar $^{\mathrm{b}} \quad$ Wamda Goreal $^{\mathrm{b}}$ \\ Jill Bunney ${ }^{b}$ Ritesh Parajuli ${ }^{b}$ \\ aUniversity of California Irvine Medical Center, Department of Medicine, Division of \\ Hematology Oncology, Orange, CA, USA; bUniversity of California Irvine Medical Center, \\ Department of Pathology, Orange, CA, USA
}

\section{Keywords}

Chemoradiation · Combined modality · Docetaxel · Head and neck cancer · Nasopharyngeal carcinoma

\begin{abstract}
Sinonasal undifferentiated carcinoma (SNUC) is a rare, poorly differentiated and aggressive malignancy of the nasal cavity and paranasal sinuses first reported by Frierson et al. in 1986 with less than 300 known cases reported since then. Due to the rarity and aggressive nature of the disease, there is a lack of consensus regarding optimal management in these patients. Treatment decisions have mostly been guided by a small number of cases series and can vary widely between institutions. In this unique case presentation, we review a case of sinonasal undifferentiated carcinoma in a young Hispanic male reviewing the literature on a rare disease, in order to elucidate effective treatment options for improved future outcomes. Based off of literature review and prior case series, the multiple modality approach should result in the best possible outcome for this rare and aggressive disease. In this specific case of a young Hispanic male with Stage IVB SNUC, we proceeded with Neo-adjuvant TPF (Docetaxel, cisplatin and fluorouracil) with effective results, followed by Cisplatin and concurrent radiation once the
\end{abstract}


patient had interval progression, and was deemed unresectable. Given the rarity and complexity of this disease, a prospective randomized controlled study should eventually be pursued to properly determine the most effective mode and combination of therapies. At this time treatment can only be based on reported case series and a small number of retrospective studies, and therefore it is important to continue to evaluate different institutions' methods of treatment.

\section{Introduction}

Sinonasal undifferentiated carcinoma (SNUC) is a rare, poorly differentiated and aggressive malignancy of the nasal cavity and paranasal sinuses first reported by Frierson et al. in 1986 with less than 300 known cases reported since then [1, 2]. It has an estimated incidence of 0.02 per 100,000 [3]. Due to the rarity and aggressive nature of the disease, there is a lack of consensus regarding optimal management in these patients. Treatment decisions have mostly been guided by a small number of cases series and can vary widely between institutions [4]. In this unique case presentation, we review a case of sinonasal undifferentiated carcinoma in a young Hispanic male reviewing the literature on a rare disease, in order to elucidate effective treatment options for improved future outcomes.

\section{Case Presentation}

Mr. T.V. is a 30-year-old Spanish speaking male with no significant past medical history, initially presenting to the ER with three weeks of right orbital and frontal headaches. His symptoms progressed to include right-sided ptosis, photophobia, nausea, vomiting, rightsided facial numbness, and intermittent anosmia. He underwent imaging in the ER with a head CT which was significant for a $4.7 \mathrm{~cm} \times 4.1 \mathrm{~cm} \times 3.9 \mathrm{~cm}$ ethmoid/sphenoid sinus mass. ENT performed an urgent biopsy confirming a poorly differentiated malignant neoplasm with positive pankeratin staining, consistent with carcinoma (Fig. 3). Tumor cells were negative for markers of neuroendocrine differentiation and squamous differentiation. The patient was p63 and EBV negative, with findings most consistent with sinonasal undifferentiated carcinoma. MRI Brain demonstrated a $5.2 \mathrm{~cm}$ AP T2-intermediate, enhancing, diffusion restricting mass centered in the ethmoid sinuses with extension into the nasal cavity, stenosis of bilateral orbital apices, encasement of bilateral optic nerves, and mass effect in the bilateral medial rectus muscles (Fig. 1). A CT chest/abdomen/pelvis was negative for metastatic disease. The patient was staged at IVB, and the case was subsequently discussed at a Multidisciplinary tumor board given the complex nature of the case. Due to the proximity of critical structures, the patient was deemed unresectable at the time. Given the large tumor burden and locally advanced disease, the decision was made to proceed with Neo-adjuvant chemotherapy, with the eventual plan for surgical resection with additional chemotherapy and radiation as needed. The patient was initiated on Docetaxel/Cisplatin/5 FU (TPF) the same week, and completed 3 cycles without complication. The patient noted significant clinical improvement in vision, diplopia and headaches during this period. The 4th cycle was completed but given without Docetaxel due to hepatotoxicity.

Repeat MRI Brain in late August 2018 showed an interval decrease in the size of the previously described sinonasal lesion now measuring approximately $4.2 \times 4.1 \times 3.2 \mathrm{~cm}(\mathrm{AP}, \mathrm{TR}$, SI), previously $5 \times 4.5 \times 4.5 \mathrm{~cm}(\mathrm{AP}, \mathrm{TR}, \mathrm{SI})$. Aspects of the mass lesion had improved 
significantly with decreased extension into the left medial orbital wall, left orbital apex and left posterior ethmoidal air cells and sphenoidal sinus, and there was near complete resolution of the mass effect on the left optic nerve and bilateral medial rectus muscles (Fig. 2). Unfortunately, the patient returned to the ER in late September 2018 with symptoms of a headache associated with nausea and vomiting. Initial CT Head showed mild interval increase, but an MRI in the next couple weeks showed a more substantial interval increase in size of sinonasal tumor with progression of intracranial tumor extension now involving the cavernous sinuses and frontal fossae. The initial Neurosurgical plan for resection was aborted in setting of such aggressive progression seen on imaging. Most recently, the patient was initiated on concurrent chemo-radiation with Cisplatin, with an interval MRI brain showing again an interval decrease in the size of his sinonasal tumor with diminished mass effect on the orbital contents, cavernous sinuses and frontal fossae.

\section{Discussion}

SNUC is a poorly differentiated and aggressive disease with limited consensus on optimal management due to the rarity of the disease. The disease is characterized by a high mitotic rate, tumor necrosis, prominent vascular permeation, and absence of glandular differentiation. It is immunohistochemically distinct from other sinonasal malignancies such as lymphoma, neuroendocrine carcinoma, mucosal melanoma, nasopharyngeal carcinoma, and olfactory neuroblastoma. Cytokeratin staining is positive while staining for leucocyte common antigen (LCA), S-100 protein, vimentin, in situ hybridization for Ebstein-Barr encoded RNA (EBER), synaptophysin and calretinin are generally negative [5]. Tumors are typically advanced at presentation with $70-100 \%$ of tumors being T4 and $10-30 \%$ of tumors having involved neck nodes [6]. Long term survival rates range from $20-40 \%$ with tumor stage being an independent predictor of survival [4, 7]. Symptoms are usually of short duration (weeks to months) contrasting with the gradual onset associated with other sinonasal tract neoplasms.

Our case demonstrates the use of TPF followed by concurrent Cisplatin with radiation in a young otherwise healthy individual diagnosed with locally advanced, unresectable SNUC. Studies have shown that an aggressive multimodality approach involving combinations of surgery, chemotherapy, and/or radiation is recommended given the high likelihood of local recurrence, generally advanced stage at presentation, and proximity to critical structures $[4,5$, 8]. Trimodality was evaluated by Mourad et al. in single institution retrospective study of 18 patients with sinonasal undifferentiated carcinoma. Neoadjuvant chemotherapy included TPF every 3 weeks for 2 to 3 cycles. In all patients receiving concurrent chemoradiation, cisplatin was used at a dose of $100 \mathrm{mg} / \mathrm{m}^{2}$ every 3 weeks for 3 cycles. The trimodality approach provided 83\% Local Control (LC) and 33\% Distant Metastases-Free (DM) survival whereas other modalities provided 50\% LC and 33\% DM-free survival. In Guo et al., 41 patients with Stage III or IV nasopharyngeal cancer were treated with TPF, with limited adverse effects, so the regimen was deemed a safe and effective way to treatment advanced disease. Morand et al. performed a case-series, systematic review and meta-analysis of the data involving treatment modalities for patients with SNUC, evaluating a total of 390 patients. $80 \%$ of patients presented with a T4 tumor and $16 \%$ with nodal metastases at diagnosis. Single modality treatment (surgery or radiation alone) was associated with reduced survival compared to double modality (surgery and radiation or chemoradiation). In this specific study it was noted that trimodality was not superior to double modality $[5,8]$. 


\section{Conclusion}

Based off of literature review and prior case series, the multiple modality approach should result in the best possible outcome for this rare and aggressive disease [4, 5, 9]. In this specific case of a young Hispanic male with Stage IVB SNUC, we proceeded with Neoadjuvant TPF with effective results, followed by Cisplatin and concurrent radiation once the patient had interval progression, and was deemed unresectable. Given the rarity and complexity of this disease, a prospective randomized controlled study should eventually be pursued to properly determine the most effective mode and combination of therapies. At this time treatment can only be based on reported case series and a small number of retrospective studies, and therefore it is important to continue to evaluate different institutions' methods of treatment.

\section{Statement of Ethics}

The authors have no ethical conflicts to disclose.

\section{Disclosure Statement}

The authors have no conflicts of interest to declare.

\section{References}

1 Frierson HF Jr, Mills SE, Fechner RE, Taxy JB, Levine PA. Sinonasal undifferentiated carcinoma. An aggressive neoplasm derived from schneiderian epithelium and distinct from olfactory neuroblastoma. Am J Surg Pathol. 1986 Nov;10(11):771-9.

2 Gamez ME, Lal D, Halyard MY, Wong WW, Vargas C, Ma D, et al. Outcomes and patterns of failure for sinonasal undifferentiated carcinoma (SNUC): The Mayo Clinic Experience. Head Neck. 2017 Sep;39(9):1819-24.

3 Chambers KJ, Lehmann AE, Remenschneider A, Dedmon M, Meier J, Gray ST, et al. Incidence and survival patterns of sinonasal undifferentiated carcinoma in the United States. J Neurol Surg B Skull Base. 2015 Mar;76(2):94-100.

4 Mourad WF, Hauerstock D, Shourbaji RA, Hu KS, Culliney B, Li Z, et al. Trimodality management of sinonasal undifferentiated carcinoma and review of the literature. Am J Clin Oncol. 2013 Dec;36(6):584-8.

5 Morand GB, Anderegg N, Vital D, Ikenberg K, Huber GF, Soyka MB, et al. Outcome by treatment modality in sinonasal undifferentiated carcinoma (SNUC): A case-series, systematic review and meta-analysis. Oral Oncol. 2017 Dec;75:28-34.

6 Tanzler ED, Morris CG, Orlando CA, Werning JW, Mendenhall WM. Management of sinonasal undifferentiated carcinoma. Head Neck. 2008 May;30(5):595-9.

7 Kuan EC, Arshi A, Mallen-St Clair J, Tajudeen BA, Abemayor E, St John MA. Significance of Tumor Stage in Sinonasal Undifferentiated Carcinoma Survival: A Population-Based Analysis. Otolaryngol Head Neck Surg. 2016 Apr;154(4):667-73.

8 Kuo P, Manes RP, Schwam ZG, Judson BL. Survival Outcomes for Combined Modality Therapy for Sinonasal Undifferentiated Carcinoma. Otolaryngol Head Neck Surg. 2017 Jan;156(1):132-6.

9 Bhasker S, Mallick S, Benson R, Bhanuprasad V, Sharma A, Thakar A. A multimodality approach to sinonasal undifferentiated carcinoma: a single institute experience. J Laryngol Otol. 2017 Jan;131(1):19-25. 


\section{Case Reports in Oncology}

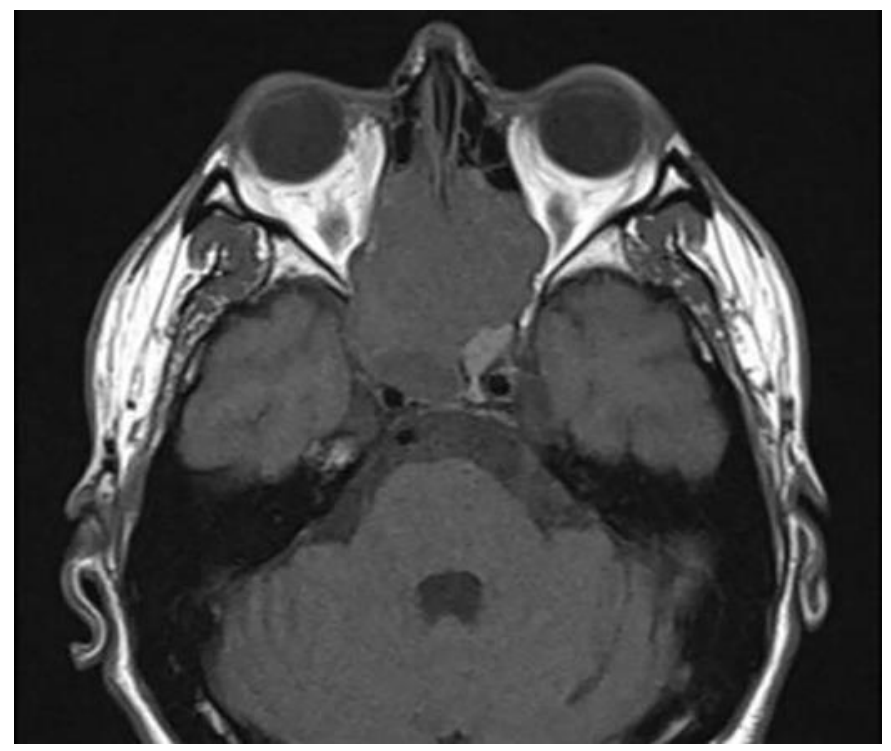

Fig. 1. MRI orbit $5 / 2018.5 .2 \times 4.3 \times 4.2 \mathrm{~cm}(\mathrm{AP}, \mathrm{TR}, \mathrm{SI}) \mathrm{T} 1 / \mathrm{T} 2$ isointense mass lesion with mildly heterogeneous enhancement and diffusion restriction centered at posterior ethmoidal air cells, and extension into posterior cerebral nasal cavity including superior turbinates, sphenoidal sinuses, bilateral orbital medial walls and anterior cranial fossa.

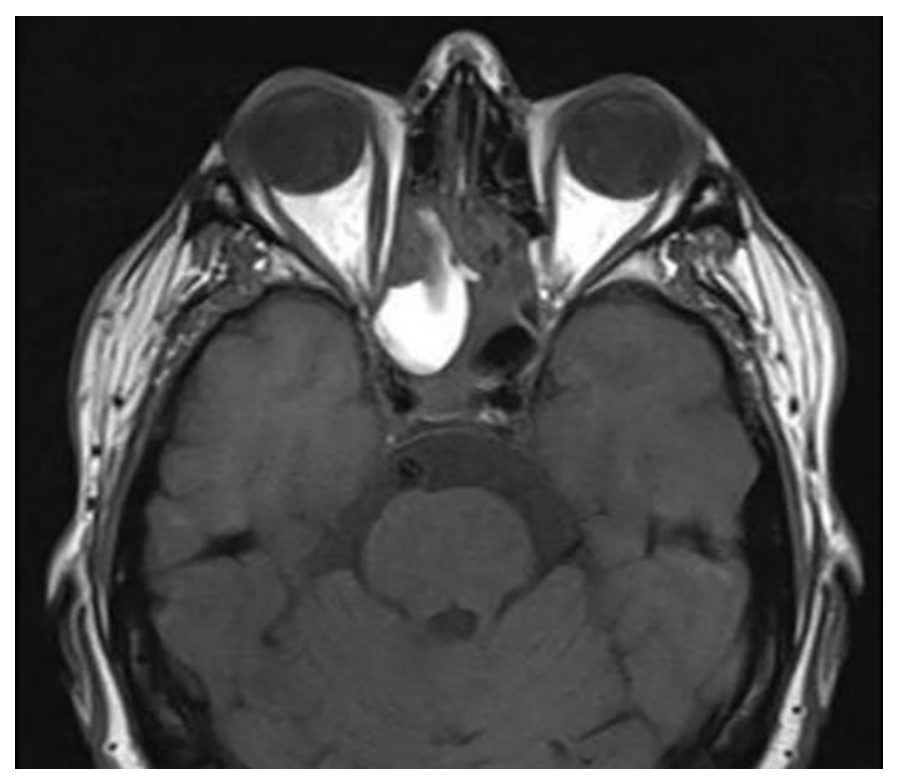

Fig. 2. MRI Orbit $8 / 2018$. Since $05 / 30 / 2018$, interval decrease in the size of the previously described sinonasal lesion now measuring approximately $4.2 \times 4.1 \times 3.2 \mathrm{~cm}$ (AP, TR, SI), previously $5 \times 4.5 \times 4.5 \mathrm{~cm}$ (AP, TR, SI). Left aspect of the mass lesion has decreased significantly with decreased extension into the left medial orbital wall, left orbital apex and left posterior ethmoidal air cells and sphenoidal sinus. Near complete resolution of the mass effect on the left optic nerve and bilateral medial rectus muscles. 


\section{Case Reports in Oncology}

\begin{tabular}{l|l}
\hline Case Rep Oncol 2019;12:199-204 \\
\hline DOI: 10.1159/000496496 & $\begin{array}{l}\text { @ 2019 The Author(s). Published by S. Karger AG, Basel } \\
\text { www.karger.com/cro }\end{array}$ \\
\hline
\end{tabular}
www.karger.com/cro

Barber et al: Treatment of Sinonasal Undifferentiated Carcinoma with TPF and Concurrent Chemo-Radiation: Case Report and Literature Review
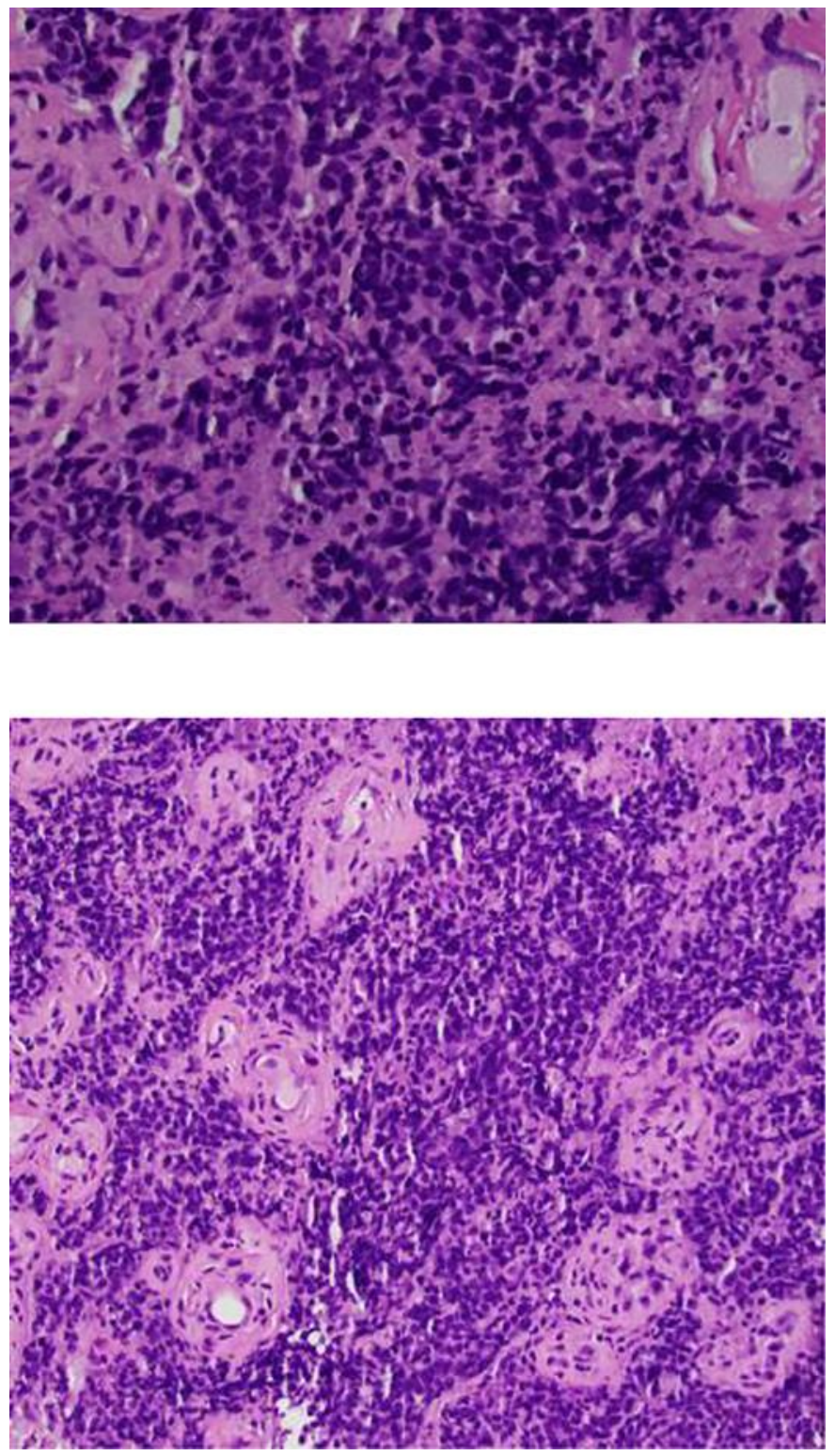

Fig. 3. Pathology slides for Patient TV confirming Sinonasal Undifferentiated Carcinoma. 